\title{
Electrodynamics of metallic photonic crystals and problem of left-handed materials
}

\author{
A. L. Pokrovsky and A. L. Efros \\ Department of Physics, University of Utah, Salt Lake City UT, 84112 USA
}

\begin{abstract}
An analytical theory of low frequency electromagnetic waves in metallic photonic crystals with a small volume fraction of a metal is presented. The evidence for such waves has been obtained recently by experiments and computations. The cutoff frequency of these waves, $\omega_{0}$ is studied. An analytical expression for the permittivity $\epsilon$ is obtained and shown to be negative below $\omega_{0}$. If the crystal is embedded into a medium with a negative $\mu$, there are no propagating modes at any frequency. Thus, such a compound system is not a left handed material (LHM). The recent experimental results on the LHM are discussed.
\end{abstract}

In his seminal work Veselago [1] has shown that if in some frequency range both the permittivity $\epsilon$ and permeability $\mu$ are negative, the electromagnetic waves (EMW's) propagate but they have some peculiar properties. All these properties come from the fact that vectors $\mathbf{k}, \mathbf{E}, \mathbf{H}$ form a left handed rather than a right handed set. It follows that the Poynting vector and the wave vector $\mathbf{k}$ have opposite directions. The materials with these properties are called the left handed materials (LHM's).

The idea that a metallic photonic crystal (MPC) may be a technological base for the LHM [2]3] appears as a result of the computational and experimental studies of a few groups 4 w which have found the EMW's in the MPC propagating above a very low cutoff frequency. The MPC's they have considered are three- or two-dimensional lattices of thin straight metallic wires. Their discovery is very interesting and important because the waves propagate under the condition $f \sigma / \epsilon_{0} \omega \gg 1$, where $f \sigma$ is an average conductivity, $f$ being the volume fraction of a metal in the system. This propagation must be due to the MPC structure, because it would be impossible in a homogeneous medium with the conductivity $f \sigma$.

Various groups obtained different cutoff frequencies and they understood them differently. The group of Soukoulis [6] qualitatively interpreted the effect of propagation in terms of waveguide modes, while the group of Pendry [7] presented a completely original physical picture based upon a new longitudinal mode, called "plasma mode". According to Pendry et al. [7] the resulting permittivity has a plasma-like behavior

$$
\frac{\epsilon}{\epsilon_{0}}=1-\frac{\omega_{p}^{2}}{\omega(\omega+i \Gamma)}
$$

however, the "plasma" frequency contains the light velocity and has a form

$$
\omega_{p}^{2}=\frac{2 \pi c^{2}}{d^{2} \ln (d / R)}, \Gamma=\frac{\epsilon_{0} d^{2} \omega_{p}^{2}}{\pi R^{2} \sigma}
$$

where $d$ is the lattice constant, $R$ is the radius of the metallic wires, $\sigma$ is the static conductivity of the metal. The same results for $\epsilon$ and $\omega_{p}$ have been later obtained theoretically by Sarychev and Shalaev [9].

The San Diego group has accepted the "plasma model" and considered the negative $\epsilon$ at $\omega<\omega_{p}$ as one of the two crucial conditions for creation of the LHM. To obtain negative $\mu$ the split ring resonators (SRR's) are added to the MPC [3,10,11. The first observation of the negative refraction at the interface of this compound system and vacuum has been reported recently [10]. The negative refraction is the most important manifestation of the LHM.

We claim in this paper that the "plasma mode" in the MPC is in fact an EMW and that is why the experiments of the San Diego group cannot be simply understood using $\epsilon$ of the MPC and $\mu$ of the SRR's. Our paper is organized as follows. First we discuss the arguments of Pendry et al. [7] in favor of the plasma model and the theoretical approach by Smith et al. 8]. Then we derive and solve an exact dispersion equation for the cutoff frequency $\omega_{0}$ and find $\epsilon(\omega)$. The results are different from Eqs.(1,2) but $\omega_{0}$ is in a very good agreement with all computational and experimental data we are aware of. It follows that all groups discuss the same mode. The permittivity becomes negative at $\omega<\omega_{0}$. Then we show that the MPC does not support any waves if it is embedded in the medium with negative $\mu$ and discuss this result together with the experimental results of the San Diego group.

The Pendry group has proposed that the plasma mode in the MPC appears as a result of an electromagnetic renormalization of the electron mass in the second order in $1 / c^{2}$. Due to this renormalization the electron mass becomes $\approx 15$ times larger than the mass of a proton. As a result, the plasma frequency of the metal shifts down into the $\mathrm{GHz}$ range. It is known, however, that the fields can be excluded from the interaction energy and the Lagrangian can be written as a function of instantaneous velocities and coordinates of the interacting charges keeping terms of the order of $c^{-2}[12]$. This so-called Darwin Lagrangian does not contain any mass renormalization due to the interaction. 
It is also strange that one should care about both mass renormalization and plasma frequency in connection with a problem based upon Maxwell equations with a static conductivity. These equations contain neither electron mass nor plasma frequency, and the very concept of electrons is not important for them.

The interpretation of the electric permittivity [Eqs.(1,22] as given by Smith et al. [8] is in terms of conventional electrodynamics and it provides a reasonable basis for a discussion. As far as we understand, it is based upon the ansatz

$$
E_{a c}=\bar{E}+i \pi R^{2} \omega L j,
$$

where $E_{a c}$ is the electric field acting on a wire, $\bar{E}$ is the average field, $j=\sigma E_{a c}$ is the current density, and $L$ is the self-inductance of the wire per unit length. Using the ansatz (33) one can easily get Eqs.(11,22).

Now we derive an exact dispersion relation for the s-polarized EMW under the condition $f \ll 1$ in the system of infinite parallel thin straight wires ordered in a square lattice. The electric field of the wave is along the wires (z-axis), while the wave vector $\mathbf{k}$ is in the $x-y$ plane. Assume that the total current in each wire is $I_{0} \exp \left[-i\left(\omega t-\mathbf{k} \cdot \mathbf{r}_{i}\right)\right]$, where $\mathbf{r}_{i}$ is the two-dimensional radius-vector of the wire in the $x-y$ plane. The external solution for electric field $E_{z}$ of one wire with $\mathbf{r}_{i}=0$ has a form

$$
E_{z}^{i}=\left(I_{0} \mu_{0} \omega / 4\right) H_{0}^{(2)}(\omega \rho / c),
$$

where $H_{0}^{(2)}=J_{0}-i N_{0}$ is the Hankel function which decays exponentially at $\operatorname{Im} \omega<0$. Neither $J_{0}$ nor $N_{0}$ has this important property. Here and below we omit the time dependent factor. The solution is written in cylindrical coordinates $z, \rho, \phi$ and it obeys the boundary condition $B_{\phi}=(i / \omega) d E_{z} / d \rho=I_{0} \mu_{0} / 2 \pi \rho$ at $\rho=R$.

The electric field created by all wires is

$$
E_{z}(\mathbf{r})=\frac{I_{0} \mu_{0} \omega}{4} e^{i \mathbf{k} \cdot \mathbf{r}} \sum_{j} e^{i \mathbf{k} \cdot\left(\mathbf{r}_{j}-\mathbf{r}\right)} H_{0}^{(2)}\left(\frac{\omega}{c} \rho_{j}\right)
$$

where $\rho_{j}=\sqrt{\left(x-x_{j}\right)^{2}+\left(y-y_{j}\right)^{2}}$, summation is over all sites of the square lattice and the sum is a periodic function of $\mathbf{r}$.

The dispersion equation follows from the boundary condition 13 that relates the total electric field at the surface of any wire $l$ to the total current through this wire $E_{z l}=I_{0} \exp \left(i \mathbf{k} \cdot \mathbf{r}_{l}\right) / \sigma_{e f} \pi R^{2}$, where $\sigma_{e f}=2 \sigma J_{1}(\kappa R) / \kappa R J_{0}(\kappa R)$, $\kappa=(1+i) / \delta$, and $\delta$ is the skin depth. At small frequencies, when $\delta>R$ one gets $\sigma_{e f} \approx \sigma$. At high frequencies, when $\delta \leq 0.1 R$, one gets the Rayleigh formula $\sigma_{e f} \approx(1+i) \sigma \delta / R$.

Note, that the EMW exists mostly if the skin-effect in the wires is strong. Using Eqs.(11.2) one can show that $\Gamma / \omega_{p}=\delta^{2} /\left(R^{2} \ln d / R\right)$, where $\delta$ is the skin depth at $\omega=\omega_{p}$ (see also Ref. 14]). We show below that the exact solution has similar properties. That is why we mostly concentrate here on the case of the strong skin-effect.

Finally, the dispersion equation for $\omega(\mathbf{k}) \equiv(\chi-i \gamma) c / d$ has a form

$$
(\chi-i \gamma) \sum_{l, m} e^{i d\left(k_{x} l+k_{y} m\right)} H_{0}^{(2)}\left(z_{l m}\right)=\frac{4 c \epsilon_{0}}{d \sigma_{e f} f}
$$

where $z_{l m}=(\chi-i \gamma) \sqrt{l^{2}+m^{2}+(R / d)^{2}}, l$ and $m$ are integer numbers, the small term $(R / d)^{2}$ under the square root is important only when $l=m=0$. Taking real and imaginary parts of Eq.(6) one gets two equations for $\chi$ and $\gamma$.

In the continuum approximation one can substitute the summation by integration in Eq.(6) to get

$$
1-\frac{c^{2} k^{2}}{\omega^{2}}+i \frac{f \sigma_{e f}}{\epsilon_{0} \omega}=0
$$

Equation (7) describes propagation of a plane wave through a homogeneous medium with the conductivity $f \sigma_{e f}$, which is possible if $f \sigma_{e f} / \epsilon_{0} \omega \ll 1$. However, outside the continuum approximation there are propagating modes in the low frequency range $f \sigma_{e f} / \epsilon_{0} \omega \gg 1$. For these modes the fields are strongly modulated inside the lattice cell and $\mathbf{E}_{z}(\mathbf{r})$ is close to zero near each wire so that the absorption is small.

We begin with the frequency $\omega_{0}$ which is the solution of Eq.(6) at $|\mathbf{k}|=0$

$$
(\chi-i \gamma) \sum_{l, m} H_{0}^{(2)}\left(z_{l m}\right)=\frac{4 c \epsilon_{0}}{d \sigma_{e f} f}
$$


This mode is an eigenmode of the system and its frequency is the cutoff frequency for the EMW's. The numerical results for the real part of the frequency are shown in Fig. 1. One can see that $\chi$ is of the order of few units. The values of $\gamma$ are of the order of the right hand side of Eq.(8). Thus, $\chi \gg \gamma$ if $f \sigma_{e f} / \epsilon_{0} \omega \gg 1$.

In addition to the numerical solution we propose an approximation valid at very small $f$, when $|\ln f| \gg 1$. We separate the term with $l=m=0$ and substitute the rest of the sum by the integral. Then

$$
(\chi-i \gamma)\left[\frac{2 i}{\pi}\left(\ln \frac{2}{\chi \sqrt{f / \pi}}-\mathbf{C}\right)-\frac{4 i}{(\chi-i \gamma)^{2}}\right]=\frac{4 c \epsilon_{0}}{d \sigma_{e f}(\chi) f},
$$

where we assume that $\gamma \ll \chi$. Here $\mathbf{C}$ is the Euler's constant. The second term at the left hand side represents the average field $\bar{E}_{z}$ which can be found from the Maxwell equation by the following way. One can show that the average (or macroscopic) magnetic induction $\overline{\mathbf{B}}$ is zero. Then $\nabla \times \overline{\mathbf{B}}=0, \bar{\jmath}_{z}+\epsilon_{0} \partial \bar{E}_{z} / \partial t=0$, and

$$
\bar{E}_{z}=\frac{I_{0}}{i \omega \epsilon_{0} d^{2}} \text {. }
$$

The second term in the square brackets of Eq. (9) is $4 \bar{E}_{z} d / \mu_{0} I_{0} c=-4 i /(\chi-i \gamma)$. Thus, the expression in the square brackets describes deviation of the field acting on a wire from the average field and it is assumed that only one term of the sum in Eq.(8) makes this difference. This approximation is similar to the ansatz of Smith et al. [8]. The main difference between Eq.(3) and Eq.(9) which is crucial for the imaginary part of the frequency and also important for the real part, is the frequency dependence of $\sigma_{e f}$ due to the skin effect. Say, in the work by Smith et al. [3] $\delta / R \approx 7 \cdot 10^{-4}$, so that the skin effect is very strong.

Figure 1 compares our result for the real part of the cutoff frequency given by Eq.(8) with the results given by Eqs.(2.9). In these calculations we assume that $\sigma_{e f}$ is given by the Rayleigh formula so that the right hand side of Eqs.(8.90) has a form $\alpha(1-i) \sqrt{\chi}$, where $\alpha=2 c \epsilon_{0} R / f d \sigma \delta$ and $\delta$ is taken at $\omega=c / d$. One can see from Fig.11 that approximation Eq.(9) is much better than approximation Eq.(2). Both approximations coincide at small $f$ and are accurate at extremely low values of $f\left(\sim 10^{-7}\right)$ when the logarithmic term in Eqs.(2., 9 ) is very large. The computational and experimental data of Ref. [6,5] are also shown at Fig. 1 and they are in a good agreement with Eq.(8). The results of Pendry group [7] (not shown) are also in a good agreement with Eq.(8). Thus, we can make the conclusion that the San Diego group, group of Pendry and Soukoulis group discuss the same mode but at different values of parameters and that our analytical theory describes the same mode as well.

Now we find the component of electric permittivity $\epsilon(\omega)=\epsilon_{z z}$, which describes the s-polarized extraordinary waves in the uniaxial crystal. It is defined by the relation

$$
\epsilon=\epsilon_{0}+i \frac{\tilde{\sigma} d}{\chi c}
$$

where effective macroscopic conductivity $\tilde{\sigma}$ relates average current density $\bar{\jmath}_{z}$ to the average electric field $\bar{E}_{z}$ by equation $\bar{\jmath}_{z}=\tilde{\sigma} \bar{E}_{z}$. To find $\tilde{\sigma}$ we introduce an external electric field $\mathcal{E}_{z} e^{-i \omega t}$. The average field $\bar{E}_{z}$ is given by equation

$$
\bar{E}_{z}=\mathcal{E}_{z}-i \frac{I_{0} \mu_{0} c}{d(\chi-i \gamma)}
$$

where the second term is the discussed above average field created by the wires. The boundary condition on a wire now has a form

$$
\frac{I_{0} c(\chi-i \gamma) \mu_{0}}{4 d} \sum_{l, m} H_{0}^{(2)}\left(z_{l m}\right)+\mathcal{E}_{z}=\frac{I_{0}}{\pi R^{2} \sigma_{e f}} .
$$

Making use of Eqs.(12,13) one can find a relation between the current and the average field which gives both $\tilde{\sigma}$ and $\epsilon$. Finally one gets

$$
\frac{\epsilon}{\epsilon_{0}}=1-\left\{\frac{\chi}{\chi-i \gamma}-i \chi\left[\frac{\chi-i \gamma}{4} \sum_{l, m} H_{0}^{(2)}\left(z_{l m}\right)-\frac{c \epsilon_{0}}{f d \sigma_{e f f}}\right]\right\}^{-1}
$$

Assuming that the medium is transparent $(\gamma \rightarrow 0, \sigma \rightarrow \infty)$ one can get the electric permittivity from the total energy density $U$ of the electric field $U=(1 / 2) \overline{E_{z}^{2}} d(\omega \epsilon) / d \omega$. We have checked that this method gives the same result for 
$\epsilon(\omega)$. The expression in the square brackets of Eq.(14) is the dispersion equation (8). One can see that Re $\epsilon$ changes sign at $\omega=\omega_{0}$ and becomes negative at $\omega<\omega_{0}$, where $\omega_{0}$ is the root of the dispersion equation (8D). The derivative $d(\operatorname{Re} \epsilon) / d \omega$ at $\omega=\omega_{0}$ is the same as the derivative which follows from Eq.(11) at $\omega=\omega_{p}$. However, far from the root of $\epsilon$ equations (14) and (1i) differ substantially.

To find $\omega(\mathbf{k})$ one should solve Eq.(6) . For small $|\mathbf{k}|$ one can get analytical result $\omega^{2}=c^{2} k^{2}+\omega_{0}^{2}$, which is isotropic in the $x$-y plane.

Now we discuss the possibility of creation of the LHM using the negative $\epsilon$ of the MPC. Suppose that the wires are embedded into a medium with the negative magnetic permeability $\mu$. One can see that in this case the propagation of any EMW is suppressed. Indeed, instead of Eq.(16) one gets equation

$$
\sum_{j} e^{i\left(k_{x} x_{j}+k_{y} y_{j}\right)} K_{0}\left(\frac{\omega}{c_{0}} \sqrt{x_{j}^{2}+y_{j}^{2}+R^{2}}\right)=\frac{2 i}{|\mu| \omega R^{2} \sigma_{e f}},
$$

where $c_{0}=1 / \sqrt{|\mu| \epsilon_{0}}$ and $K_{0}$ is the modified Bessel function. One can see that at $|\mathbf{k}|=0$ all the terms on the left hand side of this equation are positive and real if $\operatorname{Im} \omega$ is small. Thus, if the right hand side is small, the equation cannot be satisfied. At small values of $\omega$ and $|\mathbf{k}|$ summation in the Eq.(15) can be substituted by integration. Assuming that $\operatorname{Re} \omega \gg \operatorname{Im} \omega$ one gets

$$
1+\frac{k^{2} c_{0}^{2}}{\omega^{2}}=-i \frac{\sigma_{e f} f}{\omega \epsilon_{0}}
$$

This equation does not have real solutions for $\omega(k)$. Thus, at negative $\mu$ there are no propagating modes at any frequency under the study.

This result obviously follows from the fact that $\mu \epsilon_{0}<0$ in the space between the wires. Therefore we get the plus sign in the left-hand side of Eq.(16) which forbids any EMW propagation. Thus, instead of the LHM we get a material without any propagating modes.

Now we compare this result with the theoretical idea [2,3] to obtain the LHM, where negative $\epsilon$ is created by the system of wires and negative $\mu$ is created in some other way. This idea is based upon the assumption that the negative $\epsilon$ at $\omega<\omega_{0}$ results from a "longitudinal plasma mode". It is taken for granted that its frequency is independent of magnetic properties of the system, which is usually the case for plasmons. However, the mode discussed above is not a plasma mode (see also [15]). One can show that this mode has zero average value of the magnetic induction $\overline{\mathbf{B}}$ over the unit cell. In this sense this is indeed a longitudinal mode. But the average value of the magnetic energy, which is proportional to $\overline{\mathbf{B}^{2}}$, is not zero and it is large. The physics of this mode is substantially related to the magnetic energy. That is why negative $\mu$ completely destroys this mode. It destroys also the region of negative $\epsilon$. In fact this could be predicted from the observation that $\omega_{0} \sim c / d$ becomes imaginary at negative $\mu$. Say, one can see from Eqs.(1).22) that at $\mu<0$ one gets $\omega_{p}^{2}<0$ and $\epsilon>0$ at all frequencies assuming that $\Gamma$ is small.

Thus, we have shown that the simple explanation 22,3] of the negative refraction in the compound system of the MPC and SRR's, based upon the permittivity $\epsilon$ of the MPC and the negative permeability $\mu$ of the SRR's does not work because negative $\mu$ blocks propagation of EMW's in the MPC. The propagation observed by the San Diego group might be a manifestation of the remarkable conclusion of Landau and Lifshitz (See Ref. [13] p.268) that $\mu(\omega)$ does not have physical meaning starting with some low frequency. Then, the explanation of the negative refraction in this particular system would be outside the simple Veselago scenario (see Ref. [16] as an example). In this case, to explain the negative refraction one should use a microscopic equation similar to Eq.(6) but with the SRR's included.

Finally, an analytical theory of the low frequency EMW in the two-dimensional MPC is proposed. It is shown that the propagation of the low frequency waves is possible because electric and magnetic fields in the wave are strongly inhomogeneous inside the lattice cell and that electric field is small near the wires. If the dielectric part of the MPC has a negative $\mu$, no waves can propagate through the system. We argue that the explanation of the experiment Ref. [10] is much deeper than it has been supposed before.

We are grateful to A. Zakhidov and V. Vardeny who have attracted our attention to this interesting problem and to L. P. Pitaevskii and B. L. Spivak for important discussions. The work has been funded by the NSF grant DMR-0102964.

[1] V. G. Veselago, Sov. Phys.-Solid State 8, 2854 (1967); Sov. Phys. Uspekhi 10, 509 (1968). 
[2] J. B. Pendry, Phys. Rev. Lett. 85, 3966 (2000).

[3] D. R. Smith et al., Phys. Rev. Lett. 84, 4184 (2000).

[4] D. F. Sievenpiper, M. E. Sickmiller, and E. Yablonovitch, Phys. Rev. Lett. 76, 2480 (1996).

[5] D. R. Smith et al., Appl. Phys. Lett. 65, 645 (1994).

[6] M. M. Sigalas et al., Phys. Rev. B 52, 11744 (1995).

[7] J. B. Pendry et al., Phys. Rev. Lett. 76, 4773 (1996); J. B. Pendry et al., J Phys: Condens. Matter 10, 4785 (1998).

[8] D. R. Smith et al., Appl. Phys. Lett. 75, 1425 (1999).

[9] A. K. Sarychev, V. M. Shalaev, Phys. Rep. 335, 275 (2000).

[10] R. A. Shelby, D. R. Smith, and S. Schultz, Science 292, 77 (2001).

[11] J. B. Pendry et al., IEEE Trans. Microwave Theory Tech. 47, 2075 (1999).

[12] J. D. Jackson, Classical Electrodynamics (Willey \& Sons, New York, 1998), p. 598.

[13] L. D. Landau, E. M. Lifshitz and L. P. Pitaevskii, Electrodynamics of Continuous Media (Butterworth Heinemann, Oxford, 1984), p.206.

[14] A. K. Sarychev, V. M. Shalaev, cond-mat/0103145.

[15] R. M. Walser et al., Phys. Rev. Lett. 87, 119701 (2001).

[16] M. Notomi, Phys. Rev. B 62, 10696 (2000).

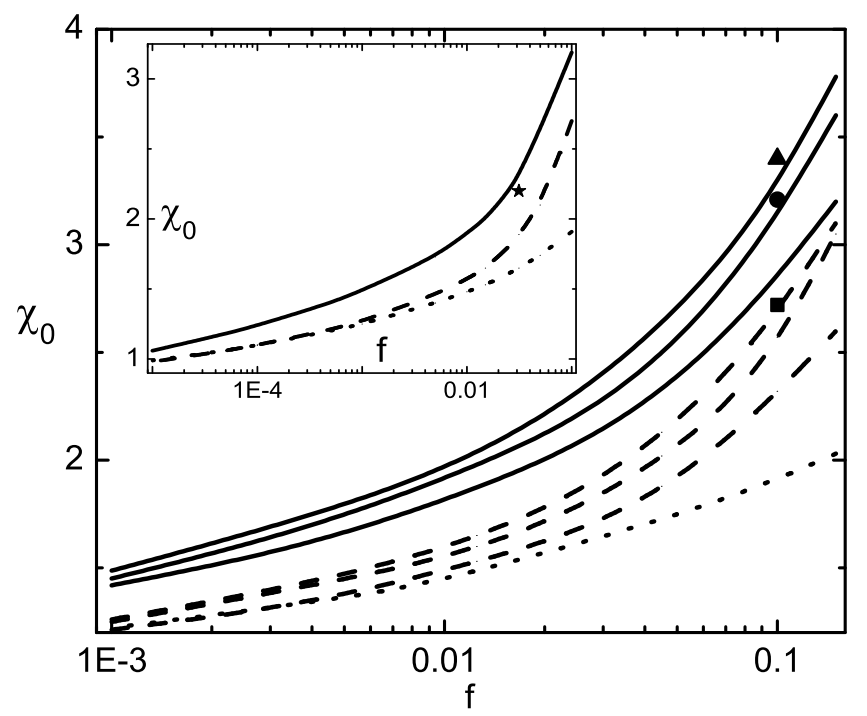

FIG. 1. The real part of the dimensionless cutoff frequency $\chi_{0}$ as a function of the volume fraction of metal $f$. Solid, dashed, and dotted lines represent solutions of Eq.(8), Eq.(9), Eq.(2) respectively. The experimental data of Ref. [3]( $\star$ ) are shown together with numerical data of Ref. [6] $(\boldsymbol{\Lambda}, \bullet, \mathbf{\square})$. On the main plot $\alpha=0.024, d=12.7 \mu m$ for upper solid, upper dashed lines, and for the point $\boldsymbol{\Lambda} ; \alpha=0.078, d=1.27 \mu \mathrm{m}$ for middle solid, middle dashed lines, and for the point $\bullet ; \alpha=0.246, d=0.13 \mu \mathrm{m}$ for lower solid, lower dashed lines, and for the point $\mathbf{\square}$. On the insert $\alpha=3.4 \cdot 10^{-4}, d=8.0 \mathrm{~mm}$. 